Kerttu Palginõmm

\title{
DIE ATTRIBUTE DER REVALER KAUFLEUTE \\ - EIN BLICK AUF DAS RETABEL DES \\ MARIENALTARES DER BRUDERSCHAFT DER SCHWARZENHÄUPTER
}

\section{MATERIELLE KULTUR AUF NIEDERLÄNDISCHEN ALTARRETABELN: EINE EINFÜHRUNG}

Die altniederländische Malerei des 15. Jahrhunderts wird definiert durch die Wiedergabe luxuriöser Texturen der dargestellten Dinge. Vorrangig richtete sich das Interesse von Kunsthistorikern dementsprechend auf die Textilien, doch auch andere Luxuswaren wurden aus der Perspektive der materiellen Kultur untersucht. Die Hauptaufgaben waren zu bestimmen, ob die dargestellten Güter die Realität exakt repräsentieren und ob diese alltäglichen Objekte symbolische Inhalte tragen. Auf beide Fragestellungen wird heutzutage mit Vorsicht geantwortet. Besonders bei gemalten materiellen Gegenständen, erzeugt die Vermittlung der Materialität 
dieser Dinge ein Spannungsfeld zwischen der Malerei und dem vermeintlichen Objekt, welches als Vorbild gedient hatte. Dabei muss dieses Vorbildobjekt in der Realität gar nicht einmal existieren, es kann sich um ein Amalgam von verschiedenen Objekten handeln dass nur im Bewusstsein des Künstlers existierte und keine Parallelen in der realen Welt fand. Wir können weder behaupten, dass diese kostbaren Gegenstände jenen Malern zur Verfügung standen, noch, dass sie die verschiedenen Dinge allgemein recht gut und aus der nahen Ansicht kannten. Genau das, was für die Vermittlung eines Objektes gewählt oder gebraucht wird, um das vermeintliche Bewusstsein des Betrachters oder des Auftraggebers zur aktivieren, kann uns Informationen bieten, die für das Verständnis des Kunstwerks wichtig sind. Die Hauptaufgabe des vorliegenden Aufsatzes besteht darin, den gemalten Rosenkranz in den Händen eines der Donatoren auf dem Revaler Retabel des Marientaltares der Bruderschaft der Schwarzenhäupter zu kontextualisieren. Bei der Analyse des dargestellten Rosenkranzes erscheint eine kombinierte Herangehensweise als angemessen: es müssen sowohl theologische, kunsthistorische, schmuckhistorische wie auch wirtschaftliche Informationen berücksichtigt werden. Die Darstellung soll im Kontext der Brügger Malerei und der Revaler Quellen interpretiert werden und zeigt, dass eine mikroskopische wie auch eine teleskopische Untersuchung eines Objekts im Rahmen der anderen Luxusgüter vielschichtige Konnotationen hervorbringen kann. Diese Konnotationen können, aber müssen nicht, dem Maler und den Donatoren zur selben Zeit verständlich gewesen sein. Die Initiative für den Habitus des Werkes kommt parallel sowohl aus Reval als auch aus Brügge und diese Anregungen fließen zusammen in ein Werk ein, das für beide Kontexte schlüssig sein kann.

\section{DAS RETABEL DES MARIENALTARES DER} BRUDERSCHAFT DER SCHWARZENHÄUPTER IM KONTEXT VON REVAL UND BRÜGGE

Das Retabel erreichte das damalige Reval wahrscheinlich im Jahre 1493 und wurde von der Großen Gilde und der Bruderschaft der Schwarzenhäupter der Dominikanerkirche St. Katharinen gespendet, um auf dem Marienaltar der Bruderschaft aufgestellt zu werden. ${ }^{1}$ Das Werk ist dem anonymen Meister der Legende der Hl. Lucia attribuiert worden - einem Brügger Maler, der im Spätmittelalter für eine breite Klientel arbeitete, die vom Mittelmeer bis zur Ostsee reichte. ${ }^{2}$ Das Retabel ist bemerkenswert wegen der Wiedergabe von Luxuswaren, die im damaligen Brügge produziert und gehandelt wurden (Abb. 1-3). Auf dem Retabel erscheinen Seiden- und Goldtücher aus Italien ${ }^{3}$, eine Verdüre aus Brügge ${ }^{4}$, ein anatolischer Teppich und eine hispano-maurische Vase ${ }^{5}$, auf die niederländische Produktion verweisende Manuskripte und kostbare Juwelen und Broschen. ${ }^{6}$ Die detailgenaue Wiedergabe von Luxusgütern hatte in Brügge eine besonders lange Tradition, die auf den Hofkünstler von Philipp dem Guten, nämlich auf Jan van Eyck, zurückgeht, der bei seiner

1 Anu Mänd, „Püha Viktor - Tallinna kaitsepühak?“, Kunstiteaduslikke Uurimusi, 12 (3-4) (2003), 10: „It.anno (14)93 do leyt her gosschalk remmlyncrade de neye taffel de to de moenken steyt vp dem altar van westen komen to der gylde vnd der swarten hoeffeden behoeff vnd de taffel steyt van lubek bet hijr der vracht $200 \mathrm{vnd} 8 \mathrm{mr}$. Kompt der gylde to betalen de helffte.
Is wat de taffel van westen bet to lubek gekostet hegen nycht dyt gelt hebbe ik wt gegeuen."

2 Mai Lumiste, ,Lucia-legendi meistri teos Tallinnas: Mustpeade vennaskonna altari autori

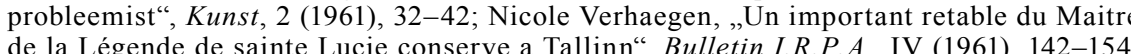
Ann M. Roberts, The Master of the Legend of Saint Lucy. A Catalogue and Critical Essay. Unveröffentlichte PhD Diss. (University of Pennsylvania, 1982), 165: Kerttu Palginõmm, Lumiste und der Problemkreis des Schaffens des Meisters der Lucialegende“, Kunstiteaduslikke Uurimusi, 22 (2014), 69.

3 Kerttu Palginõmm, „Der dem Meister der Lucialegende zugeschriebene Revaler (Tallinner) Retabel - kostbare Stoffe und ein unbekannter Meister?“, Baltic Journal of Art History, (2012), 117-141.

4 Jacqueline Versyp, De geschiedenis van de tapijtkunst de Brugge (Brussel: Paleis der Academiën, 1954), 39; Guy Delmarcel, Erik Duverger, Bruges et la Tapisserie (Bruges Mouscron: de Poortere, 1987), 20; Guy Delmarcel, Flemish Tapestry (New York: Abrams, 2000), 180; Palginõmm, „Der dem Meister der Lucialegende zugeschriebene Revaler (Tallinner) Retabel kostbare Stoffe und ein unbekannter Meister?““, 123-126.

5 Marco Spallanzani, Maioliche Ispano-Moresche a Firenze neli rinascimento (Firenze: SPES - Studio Per Ed. Scelte, 2006); Marco Spallanzani, Oriental rugs in Renaissance Florence luxury in Medieval Tallinn: the oriental rug and lusterware on the altarpiece of the Bruges Master of the St. Lucy legend“, Asjade võim = Macht der Dinge, hrsg. von Kerttu Palginõmm (Tallinn: Eesti Kunstimuuseum, 2019), 38-49.

6 Kerttu Palginõmm, Ivar Leimus, „Der Marienaltar der Bruderschaft der Schwarzenhäupter vor dem Hintergrund des Wertes der abgebildeten Luxusgüter", Kunstiteaduslikke Unrimusi, 24 (3-4) (2015), 49-85. Asjade võim, hrsg. von Kerttu Palginõmm (Tallinn: Eesti Kunstimuuseum, 2018), 66-69. 


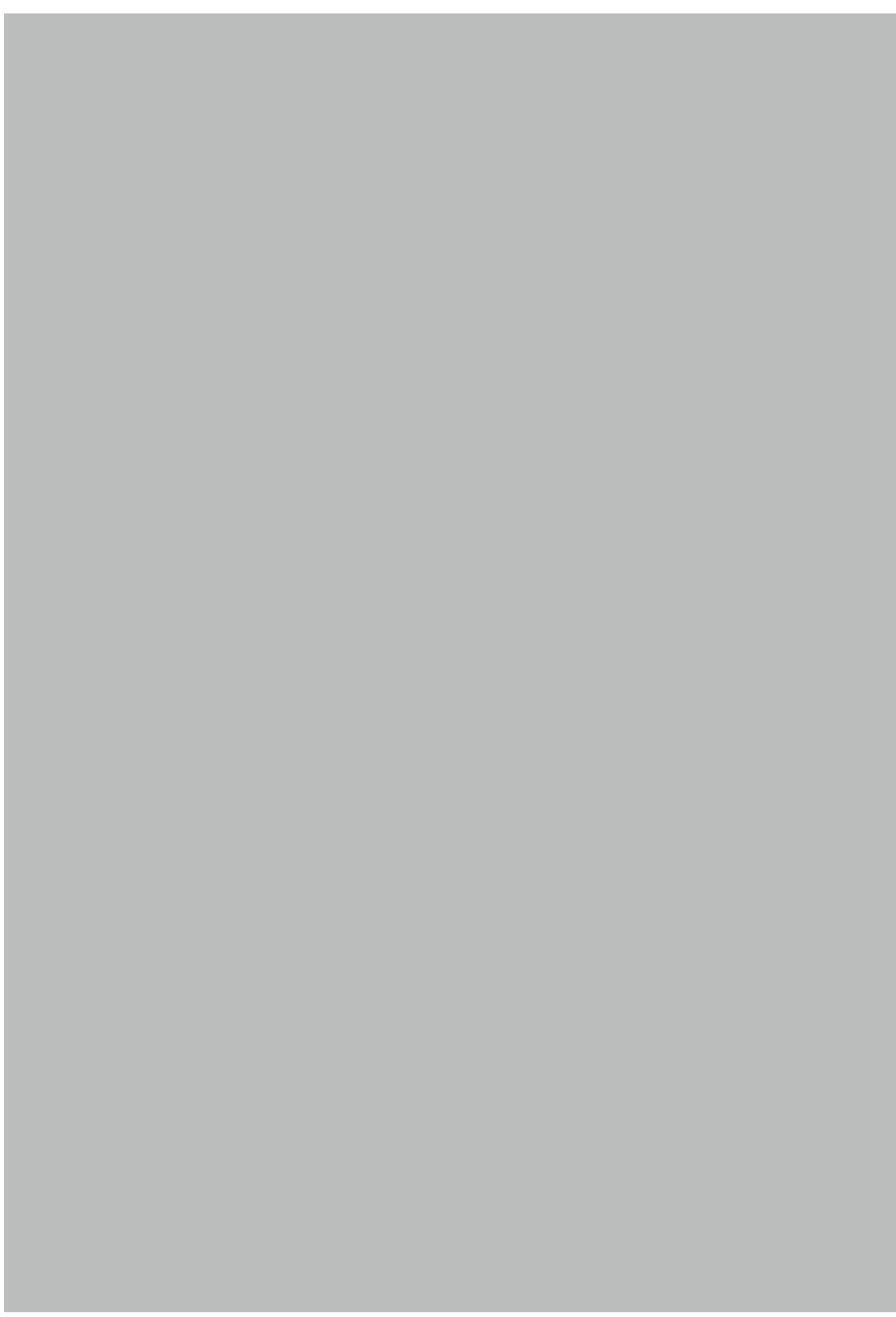

ABB. 1. MEISTER DER LEGENDE DER HL. LUCIA (ATTRIBUIERT). RETABEL DES MARIENALTARES DER BRUDERSCHAFT DER SCHWARZENHÄUPTER. GESCHLOSSENER ZUSTAND. MARIÄ VERKUUNDIGUNG. VOR 1493. OL UND TEMFERA AUF HOLZ. FLUGEL: $93 \times 95$ CM. ESTNISCHES
ABB. 2. MEISTER DER LEGENDE DER HL. LUCIA (ATTRIBUIERT). RETABEL DES MARIENALTARES DER BRUDERSCHAFT DER SCHWARZENHÄUPTER. ERSTE WANDLUNG. DOPPELTE FÜRBITTE. VOR 1493. ÖL UND TEMPERA AUF HOLZ. FLÜGEL: $93 \times 95$ CM. ESTNISCHES KUNSTMUSEUM - NIGULISTE MUSEUM. FOTO: STANISLAV STEPAŠKO.

Präzision in der Vermittlung von kostbaren Textilien ${ }^{7}$, Keramiken Metall und Glas eine besondere Meisterschaft bewiesen hatte. ${ }^{8}$ Jan van Eycks besondere Errungenschaft, und eine Errungenschaft der altniederländischen Kunst insgesamt, besteht in dem Können, die

7 Lisa Monnas, ,Silk Textiles in the Paintings of Jan van Eyck“, Investigating Jan van Eyck [papers at the Jan van Eyck Symposium held at the National Gallery on 13-14 March 1998], hrsg. von Susan Foister (Turnhout: Brepols, 2000), 147-162; Lisa Monnas, Merchants, Princes and Painters: Silk Fabrics in Italian and Northern Paintings, 1300-1550 (New Haven: Yale

8 Craig Harbison, Jan van Eyck: the play of realism (London: Reaktion, 2011). 
ABB. 3 MEISTER DER LEGENDE DER HL. LUCIA (ATTRIBUIERT). RETABEL DES MARIENALTARES DER BRUDERSCHAFT DER SCHWARZENHÄUPTER. ZWEITE WANDLUNG. SACRA IÜ̈GEL: $93 \times 95$ CM. ESTNISCHES KUNSTMUSEUM - NICUUSTE MULEUM. FOTO: STANISL STEPAŠKO.

Textur so wiederzugeben, wie sie durch Licht charakterisiert wird. ${ }^{9}$ Dabei steht im Vordergrund, wie die Textur dem Auge erscheint. ${ }^{10}$ Jan van Eycks akribische Genauigkeit blieb dabei unübertroffen. Aus der Darstellung der alltäglichen Gegenstände erwuchs für die kunsthistorische Forschung eine Methodik der „verkleideten

9 Bernhard Ridderbos, „Objects and Questions“, Early Netherlandish Paintings. Rediscovery, Reception and Research, hrsg. von Bernhard Ridderbos (Amsterdam; Los Angeles: Amsterdan University Press; The Getty Foundation, 2005), 43.

10 Monnas, Merchants, Princise and Painters, 110-111.
Symbolik", die in den Dingen einen komplizierten symbolischen Inhalt sehen wollte. ${ }^{11}$ Die darauf abzielende Kritik verwies auf die Tatsache, dass weder über den Bildungshorizont des Meisters und sehr oft auch nicht über den des Auftraggebers berichtet worden war, was es nicht erlaube, ihnen Kenntnisse über tiefgründige theologische Inhalte zuzuschreiben. ${ }^{12}$ Die Kritiker vertraten die Ansicht, die alltäglichen Gegenstände wären eigentlich aus der Umgebung des Auftraggebers oder Künstlers entnommen worden und reflektieren dementsprechend den Zeitgeschmack und die zeitgenössische Umwelt. ${ }^{13}$

Heilige Personen mit Luxusgütern $\mathrm{zu}$ inszenieren, war eine gewohnte Praxis in der niederländischen Kunst, doch die Dichte der dargestellten Güter auf dem Revaler Retabel ist in der niederländischen Malerei ohne Parallele. Deshalb eignet sich das Retabel besonders gut für die detailliertere Untersuchung von Gegenständen, die einen vielseitigen Charakter haben und sowohl aus der Nahsicht als auch der Fernsicht erforscht werden sollten.

\section{DIE ATTRIBUTE DER DONATOREN AUF DEM REVALER RETABEL}

Die Donatoren des Revaler Retabels nehmen einen wichtigen Platz im Rahmen des Retabels ein. Sie erscheinen in der zweiten Wandlung in der Szene der doppelten Fürbitte unter den Füßen der Gottesmutter und Johannes des Täufers (Abb. 4). Auf beiden Seiten unter dem Schutz der heiligen Figuren sind 15 kniende und betende Männer dargestellt. Einige von ihnen richten ihren Blick auf die Heiligen, die zentralen Figuren aber sind nach niederländischen Vorbildern in ein meditatives Gebet versunken und rufen den gläsernen Blick des Kanzlers Nicolas Rolin oder Joris van der Paeles auf den

11 Erwin Panofsky, ,Jan van Eyck's Arnolfini Portrait“, The Burlington Magazine for Connoisseurs, vol. 64, No. 372 (1934), 126.

12 Barbara C. Lane, „,Sacred versus profane“, Simiolus: Netherlands Quarterly for the History of Art, Vol. 18, No. J (19letin, Vol. LXVI, No 4 (1984), 589-590; Craig Harbion, "Fact, Symbol, Ideal: Roles for Realism in Early Netherlandish Painting" Petrus Christus in Renaissance Bruges: An Interdisciplinary Approach, hrsg. von Maryan Aisworth (New York: Metropolitan Bruges. An Interdisciplinary Approach, hrsg. von Maryan Aisworth (New York: Metropolitan

13 Jozef de Coo, ,A Medieval Look at the Merode Annunciation“, Zeitschrift für Kunstgeschichte, Bd. 44, H. 2 (1981); Ellen Callman, „Campin's Maiolica Pitcher“, The Art Bulletin, Vol. 64, No. 4 (December 1982), 629-631. 


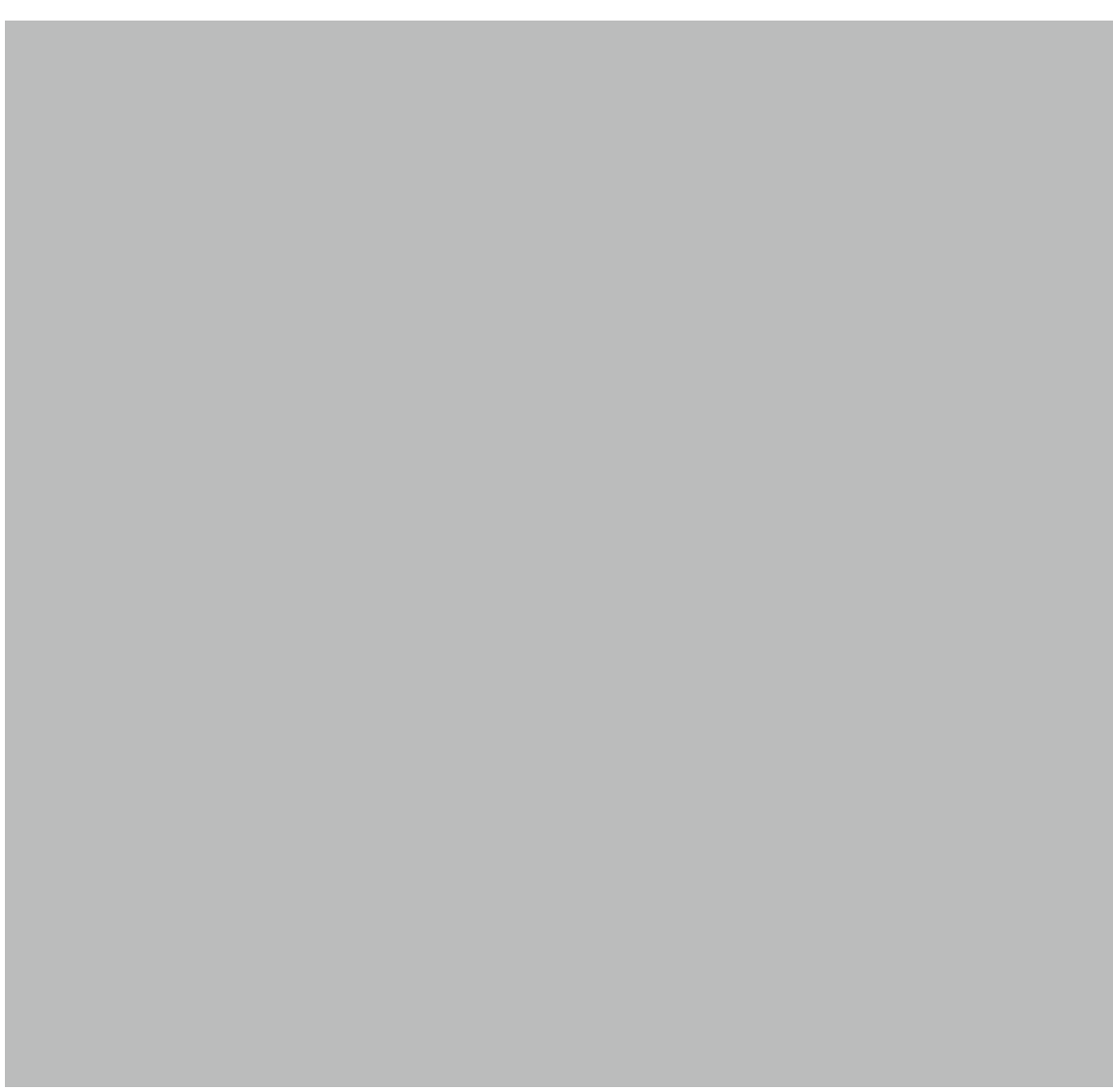

ABB. 4. MEISTER DER LEGENDE DER HL. LUCIA (ATTRIBUIERT). RETABEL DES MARIENALTARES DER BRUDERSCHAFT DER SCHWARZENHÄUPTER. ERSTE WANDLUNG. KNIENDE DONATOREN. VOR 1493. OL UND TEMPERA AUF HOLZ. MITTELTAFEL: 255,5 × 186 CM; FLUGEL: $93 \times 95$ CM. ESTNISCHES KUNSTMUSEUM - NIGULISTE MUSEUM. FOTO: STANISLAV STEPAŠKO

Altarretabeln ${ }^{14}$ des Jan van Eyck in Erinnerung. Die zentralen Figuren der 15 Männer sind auch die Malereien höchster Qualität im Rahmen des Altarwerks, weshalb man sie für Portraits hält. ${ }^{15}$ Die großen

14 Jan van Eyck. Madonna des Kanzlers von Rolin. 1435, Öl auf Holz, 66 x 62 cm, Musée du Louvre, Paris; Jan van Eyck. Madonna mit Kanonikus Joris van der Paele, 1436, 140,8 × 176, $\mathrm{cm}$, Groeningemuseum, Brügge.

15 Verhaegen, „Un important retable“, 146.

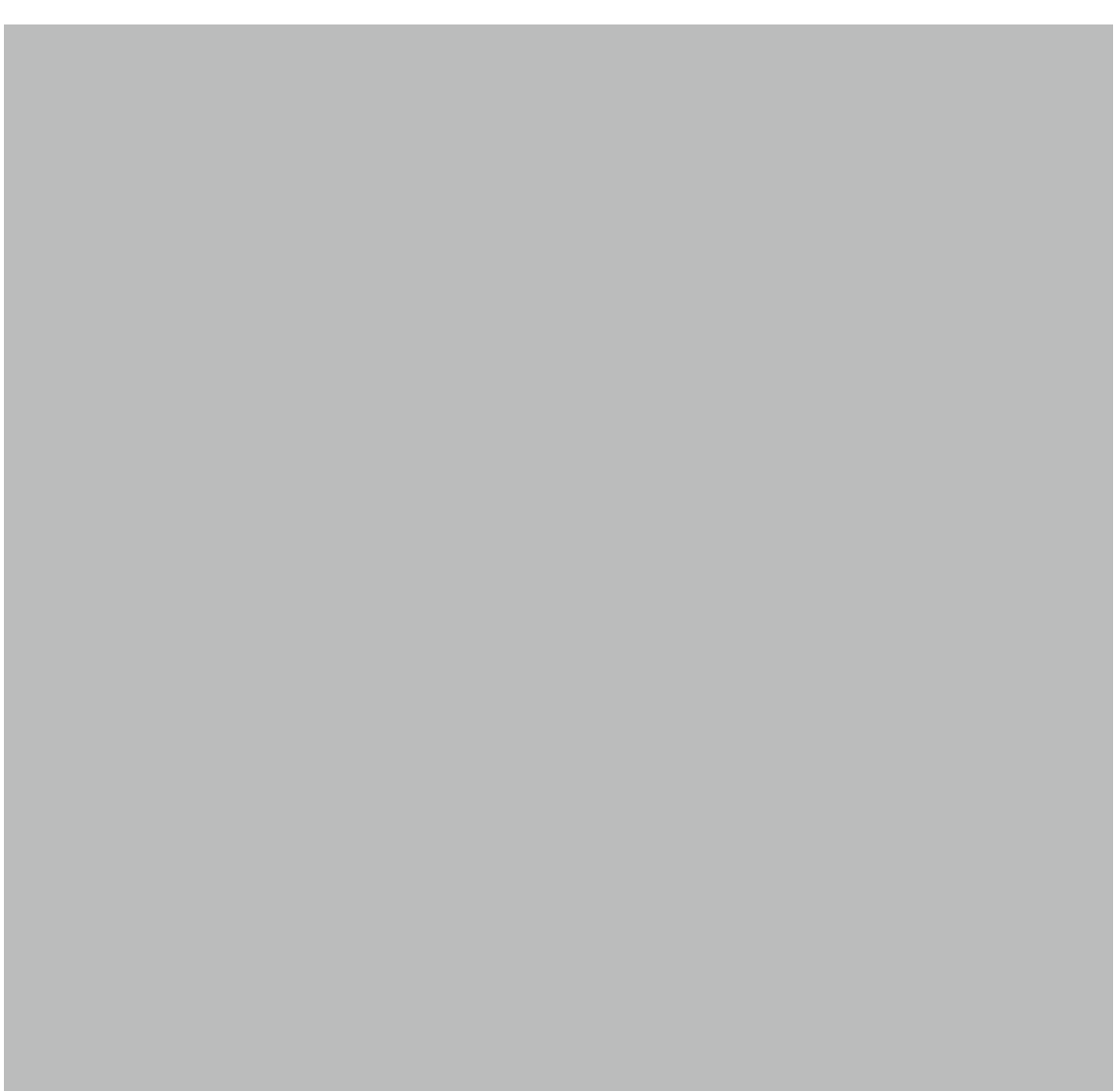

ABB. 5. MEISTER DER LEGENDE DER HL. LUCIA (ATTRIBUIERT). RETABEL DES MARIENALTARES DER BRUDERSCHAFT DER SCHWARZENHÄUPTER. ERSTE WANDLUNG. ROSENKRANZ IN HÄNDEN EINES KAUFMANNS. VOR 1493. OL UND TEMPERA AUF HOLZ. MITTELTAFEL 255,5 × 186 CM; FLÜGEL: 93 × 95 CM. ESTNISCHES KUNSTMUSEUM - NIGULISTE MUSEUM.

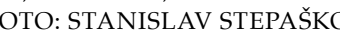

Qualitätsschwankungen auf dem Retabel lassen uns glauben, dass mehrere Künstlerhände der Werkstatt hier im Spiel waren. ${ }^{16}$

Die Donatoren tragen Wämser und Mäntel die aus verschiedenfarbigen, qualitativen Wollstoffen genäht und mit schmalen Gürteln ergänzt sind. An den Gürteln befinden sich Geldtaschen und Geldbeutel. Diese kennzeichnen sie als vornehme

16 Juhan Maiste, „Michel Sittow - Tallinna mees“, Eesti kunsti ajalugu, 2: 1520-1770, hrsg. von Krista Kodres (Tallinn: Eesti Kunstiakadeemia, 2005). 
und vermögende Bürger und nehmen weiterhin Bezug auf ihre Tätigkeit als Händler. Sie sind nicht nur als Identifikationsmittel für die Auftraggeber zu interpretieren, sondern dienen als Zeugen von einem allgemeinen Wohlstand und können als Verweise auf wichtige Tugenden wie Mildtätigkeit (Caritas) angesehen werden. Die Kaufleute tragen unter anderem auch Siegelringe, die im Kaufmannsgeschäft eine Rolle spielten und für das Abschließen von Verträgen eingesetzt wurden. Die Siegelringe verkörpern und stellen Machtverhältnisse und Autorität dar. Sie trugen Monogramme, die in dem Indentifikationsprozess eine Rolle spielen. Auf dem Revaler Retabel sind leider keine erkennbaren Monogramme erhalten, was sehr wahrscheinlich durch die Tatsache bedingt wird, dass der Meister der Legende der H1. Lucia sich dessen nicht bewusst ist und nur einem traditionellen Schema eines mittelalterlichen Hansekaufmanns und seines Accessoires folgt.

An den Gürteln befestigte Geldtaschen wurden in der Malerei der Vorgänger und der Zeitgenossen des Meisters prominent dargestellt. Man erinnere sich in diesem Zusammenhang an den Kanzler Rolin, der auf dem Retabel Jan van Eycks zuerst mit einem großen Geldbeute dargestellt werden sollte, sich aber später wohl gegen dieses Motiv entschied, wie dies die Unterzeichnungen bezeugen. ${ }^{17}$ Nichtdestotrotz trägt der Kanzler, der neben seiner Rolle als Ratgeber noch als Schatzmeister für die Finanzen im Burgunderreich zuständig war ${ }^{18}$, seine Geldtasche ${ }^{19}$ auf anderen Portraits ${ }^{20}$ mit der Ausnahme des Beaune Polyptychons ${ }^{21}$. Die Geldtaschen und Geldbeutel in der Rolle eines Attributs liefern einen gewichtigen Grund, den Donatoren eine merkantile Rolle zuzusprechen. Die dargestellten Accessoires, können auch einen Beleg dafür liefern, dass der Maler, von Reval weit entfernt, über die Auftraggeber gut informiert war und sie mit entsprechenden

17 Elisabeth Dhanens, Hubert und Jan van Eyck (Antwerpen: Mercatorfonds, 1980), 279, Abb. 173, Abb. 174 .

18 Richard Vaughan, Philip the Good: the apogee of Burgundy (Woodbridge; Rochester: Boydell Press, 2011), 101

19 Rogier van der Weyden. Widmungsminiatur der Chroniques de Hainaut, Bibliothèque Royale des Roman de Girart de Roussillions, ca. 1450, Nationalbibliothek, Wien, Cod. 2549, fol. 6.

20 John L. Ward, „Disguised Symbolism as enactive Symbolism in Van Eyck's paintings“, Artibus et Historiae, 29 (1994), 9-53, 37.

21 Rogier van der Weyden. Polyptychon des Jüngsten Gerichts, Öl auf Holz, $215 \times 560 \mathrm{~cm}$, Musée de l'Hôtel-Dieu, Beaune.

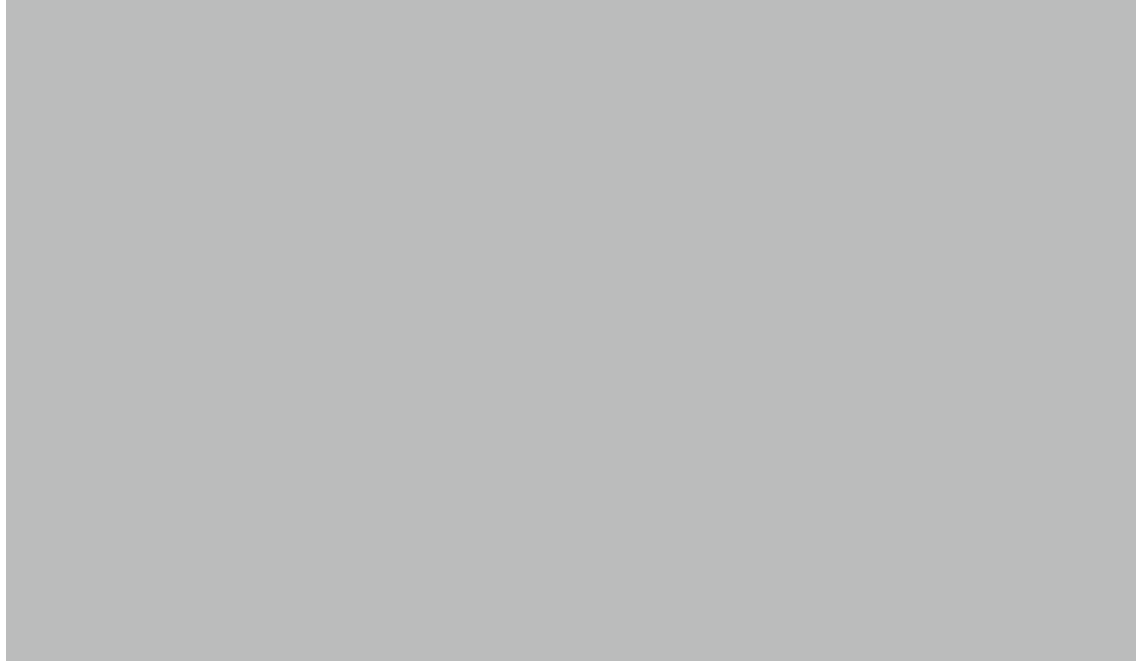

ABB. 6. BEUTEL AUS LEDER. 14.-15. JAHRHUNDERT. 21,5 × 17,5 CM. TLM. 27426:1 A. TALLINNER STADTMUSEUM. FOTO: STANISLAV STEPAŠKO.

Attributen versah. Die dargestellten Geldtaschen und -beutel stellten selbstverständlich keine einzig den Kaufleuten von Reval zugehörigen Gegenstände dar, auch wenn wir vermuten müssen, dass die zentralen Figuren für das Retabel portraitiert wurden. In der Kollektion des Tallinner Stadtmuseums ist ein Geldbeutel erhalten, der ein Beispiel für einen wahrhaftig einem Revaler Bürger gehörenden Beutel darstellt (Abb. 6). ${ }^{22}$ Dennoch muss im Auge behalten werden, dass der Meister den Attributen eine besondere Aufmerksamkeit schenkt und sie detailliert wiedergibt - sie sind mit kleinen Nieten, Metallverschlüssen und dekorativen Schnüren ausgestattet. Diese Aufmerksamkeit deutet auf die Wichtigkeit der Attribute bei dem Identifikationsprozesse der Schenker hin, deren Beschäftigung ganz klar zur Geltung gebrach wird. In ihrer Ausführung ist die lederne, mit silbernen Verschlüssen und einer löwenkopfähnlichen Applikation versehene Geldtasche mit einer ähnlich gestalteten Gürteltasche auf dem Mérode Triptychon des

22 Beutel aus Leder. 14.-15. Jahrhundert. $21,5 \times 17,5 \mathrm{~cm}$. Stadtmuseum Tallinner Stadtmuseum. 
Meisters von Flémalle ${ }^{23}$ vergleichbar, die vom Spender getragen wird. Nicolas Rolin, wenn auch von niederer Herkunft ${ }^{24}$, war der wichtigste Hofbeamte seiner Zeit, doch die Donatoren ${ }^{25}$ des Mérode Triptychons, vergleichbar wie die Donatoren des Revaler Retabels, gehörten dem gehobenem Bürgertum an, präziser der Kaufmannschaft. Bei den Figuren der Spender des Revaler Retabels lenkt noch ein weiteres ihrer Attribute Aufmerksamkeit auf sich - es handelt sich um einen Rosenkranz mit gelben Gebetsperlen, von denen elf sichtbar sind (Abb. 5).

\section{ROSENKRÄNZE IN DER ALTNIEDERLÄNDISCHEN MALEREI DES 15. JAHRHUNDERTS}

Gebetsschnüre respektive Rosenkränze wurden im Spätmittelalter aus verschiedensten Materialien hergestellt wie Koralle, Kristall, Bernstein, aber auch aus Bein, Horn, Muscheln, Gagat oder auch Glas und Holz, an ihnen waren oft noch Anhänger angebracht worden wie Kreuze, Reliquienkapseln, Medaillen, Pilgerzeichen usw. ${ }^{26}$ Seit dem 13. Jahrhundert sind spezialisierte Rosenkranzhersteller bekannt, die sich auch in eigenständigen Gilden organisierten. ${ }^{27}$ Der Rosenkranz in seiner klassischen Form bestand aus 50 Ave-Maria-Perlen abwechselnd mit Paternoster-Perlen und einem abschließenden Objekt. Der Zehner, der um das Jahr 1500 verbreitet wurde, beinhaltete gewöhnlich zehn

23 Meister von Flémalle (Robert Campin?), Triptychon mit der Verkündigung (MérodeTriptychon), Mitteltafel 64,1 × 63,2 cm, Flügel 64,5 × 62,0, Cloisters Collection, Metropolita Museum of Art, New York.

24 Obwohl Kanzler Rolin das höchste Amt am Hof Phillipps des Guten innehatte, stammte er aus bescheidenen Verhältnissen: Siehe: Laura Gelfand, „Piety, Nobility and Posterity: Wealth 2015), http:/Www jhate [03.08.2015].

25 Die Namen der Auftraggeber für das Mérode Triptychon sind nicht quellensicher bewiesen. Doch wird von den auf dem Retabel vorkommenden Wappen ausgehend, vermutet, dass sie den Mechelener Imbrechts oder den Kölner Engelbrechts angehörten. Es ist möglich, dass der der Weyden hrsg. von Stephan Kemperdick (Ostfildern: Hatje Cantz, 2008), 197-199.

26 Anna Beatriz Chadour-Sampson, „Rosenkränze - Pretiosen der Andacht. Schmuckhistorische Aspekte“, Der Rosenkranz: Andacht, Geschichte, Kunst [Ausstellung ,Zeitinseln - Ankerperlen, Geschichten um den Rosenkranz" Museum Bruder Klaus Sachseln 25. Mai bis 26. Oktobe 2003] (Bern: Benteli, 2003), 471 .

27 Chadour-Sampson, „Rosenkränze - Pretiosen der Andacht“, 472. Ronald W. Lightbown, Mediaeval European jewellery (London: Victoria \& Albert Museum, 1992), 343.
ABB. 7. MEISTER VON FLÉMALLE (ROBERT CAMPIN?). TRIPTYCHON MIT DER VERKÜNDIGUNC (MÉRODE-TRIPTYCHON). UM 1427-1432. ÖL AUF HOLZ. MITTELTAFEL: 64,1 × 63,2 CM; FLÜGEL GROUP S.P.A.

Perlen, einen Tragering am Anfang und ein Abschlussobjekt. ${ }^{28}$ Diese gehörten vornehmlich Männern. ${ }^{29}$

Die Rosenkränze finden sich öfters auf den Darstellungen der Donatoren in der altniederländischen Malerei, besonders au den Retabeln und den Miniaturen. Der Hintergrund dafür war die Frömmigkeitspraxis der Zeit, bei der das meditative Gebet im Vordergrund stand und als dessen Ausdrücke Manuskripte

28 Rosenkränze und Gebetsschnüre: die Sammlung Weihbischof Heinrich Janssen. 28 Rosenhranze und Gebersschnire: die Sammlung Weihbischof Heineich Janssen. Goch: Völcker, 2013), 18. 29 Anne Margareet W. As-Vijvers, ,Weaving Mary's Chaplet: The Representation of the Rosary in Late Medieval Flemish Manuscript Illumination", Weaving, Veiling and Dressing: Textiles and their Metaphors in the Late Middle Ages, hrsg. von Barbara Baert, Kathryn Rudy (Turnhout: Brepols, 2007), 71. 
und Gebetsschnüre angesehen werden können. ${ }^{30}$ Wir finden die Wiedergabe dieser Güter sowohl bei adligen wie auch bürgerlichen Donatoren, sowohl bei Männern wie bei Frauen. So findet sich ein Rosenkranz auf dem berühmten Mérode Triptychon des Meisters von Flémalle ${ }^{31}$, für dessen Donatoren die Kölner Kaufmannsfamilie Engelbrecht/Ymbrecht gehalten wurde (was aber nicht durch Quellen bestätigt wird) (Abb. 7). ${ }^{32}$ Der Rosenkranz aus Korallen in den Händen der Ehefrau mit einem Abschlussobjekt als Christophorus, also "Christusträger", wurde auf den Kinderwunsch des Paares zurückgeführt. ${ }^{33}$ Ein Rosenkranz war ein typischer Geschenk für eine junge Ehefrau, um ihre devote Haltung zu stärken. ${ }^{34}$

Ein Rosenkranz aus Koralle wird dargestellt in den Händen eines Mannes auf dem Retabel von Hans Memling ${ }^{35}$, dessen schwarze Kleidung mit Ärmeln aus Damast und mit einem Pelzkragen besetzt ist. ${ }^{36}$ Sein Rosenkranz ist besonders, weil er mit einem sogenannten Duftkugel endet ${ }^{37}$ - es war gewöhnlich, Gewürze und Kräuter in einer Kapsel zu verschließen, was wieder auf den Status des Donators Bezug nimmt und eine heilende und beschützende Wirkung verspricht.

Den Zehner treffen wir bei Portraits von Adligen an $^{38}$, wie bei Philippe de Croÿ, der ein Höfling von Philipp dem Guten von Burgund war und dessen Vorliebe für luxuriöse Manuskripte er teilte ${ }^{39}$

30 Bret L. Rothstein, Sight and Spirituality in Early Netherlandish Painting (Cambridge: Cambridge University Press, 2005), 95-96.

31 Meister von Flémalle (Robert Campin?), Triptychon mit der Verkündigung (MérodeTriptychon). Um 1427-32, Öl auf Holz, Mitteltafel: $64.1 \times 63.2 \mathrm{~cm}$; Flügel: $64.5 \times 27.3 \mathrm{~cm}$, Cloisters Collection, Metropolitan Museum of Art, New York.

32 Jochen Sander, „Meister von Flémalle. Mérode-Triptychon“, Der Meister von Flémalle und Rogier van der Weyden: eine Ausstellung des Städel-Museums, Frankfurt am Main, [21.11.2008 hrsg. von Stephan Kemperdick (Ostfildern: Hatje Cantz, 2008), 199.

33 Sander, „Meister von Flémalle. Mérode-Triptychon“, 194.

34 Harbison, Jan van Eyck, 36.

35 Diptychon mit Madonna und Kind zwischen musizierenden Engeln. 1490 oder später? Flügel je: $43,3 \times 31,1 \mathrm{~cm}$. Alte Pinakothek, München.

36 Dirk De Vos, Hans Memling: das Gesamtwerk (Stuttgart: Belser, 1997), 313.

37 Ibidem.

38 Rogier van der Weyden. Diptychon mit Philippe de Crö̈, terminus ante quem 1461. Öl auf Holz. 51,5 × 33,6 cm. Koninklijk Museum voor Schone Kunsten, Antwerpen.

39 De Vos, Rogier van der Weyden: das Gesamtwerk, 305 (deshalb war auf dem wahrscheinlichen Pendant ${ }^{40}$ die Gottesmutter mit einem luxuriösen Buch dargestellt). In seinen Händen hält er eine Gebetsschnur aus Kristall oder jedenfalls aus gläsernen und hellbraunen Perlen, dessen Abschlussobjekt ein Kruzifix ist.. ${ }^{41}$ Seinen Status unterstreichen sein Dolch, der damals bei jungen Adligen in Mode war, seine schwarze Kleidung und eine feine Goldkette um seinen Hals. ${ }^{42}$

Ein interessantes Beispiel liefert noch ein Portrait ${ }^{43}$, dass Michel Sittow attribuiert wird. Es stellt einen älteren Mann dar, dessen Kleidung dem 16. Jahrhundert entspricht. ${ }^{44}$ Über einem weißen Hemd trägt er einen Wams mit Pelzbesatz (Luchs) und eine schwarze Mütze. Sowohl die Konzeption, die Maltechnik als auch die materiellen Dinge nähern dieses Portrait dem Hauptwerk von Michel Sittow an - dem Portrait ${ }^{45}$ des Diego de Guevara. ${ }^{46}$ So wie beim Diptychon des Diego de Guevara, war das Pendant des Portraits in einer Privatkollektion wahrscheinlich die Gottesmutter, an die der Donator sich mit seinem Rosenkranz aktiv betend richtet. Das Portrait ist bemerkenswert wegen der Tatsache, das dass Bild, entsprechend der Zeit des Aufenthalts von Michel Sittow (1469-1525) in Reval entstanden sein muss. ${ }^{47}$ Der in Reval gebürtige, in Brügge ausgebildete und sowohl an den Höfen von Spanien, den Niederlanden, England und in Dänemark geschätzte Portraitist, kam an seinem Lebensende wieder nach Reval zurück. ${ }^{48}$ Hier machte er Karriere und stieg in 1524 zum Ältermann der Knutsgilde auf, welche die vornehmsten

40 Rogier van der Weyden. Maria mit Kind, terminus ante quem 1461. Öl auf Holz. 50,8 $\times 33 \mathrm{~cm}$ Henry E. Huntington Library, San Marino

41 De Vos, Rogier van der Weyden, 305.

42 Ibidem.

43 Michel Sittow (?). Portrait eines Mannes mit einem Rosenkranz. Um 1520. Öl auf Holz, $33,6 \times 22,8 \mathrm{~cm}$. Privatkollektion.

44 Michel Sittow. Estonian Painter at the Courts of Renaissance Europe, hrsg. von John Oliver Hand, Greta Koppel (Washington: National Gallery of Art, 2017), Cat. 16, 92

45 Michel Sittow. Portrait eines Mannes (Diego de Guevara?). Um 1515-1518. $33.6 \times 23.7 \mathrm{~cm}$ Öl auf Holz. Andrew W. Mellon Collection, National Gallery of Art, Washington.

46 Michel Sittow. Estonian Painter at the Courts of Renaissance Europe, Cat. 16, 92-94.

47 Ibidem, Cat. 16, 94.

48 Matthias Weniger, „An Artist's Career between the Hanseatic City of Tallinn and the Princely Courts of Europe”, Michel Sittow. Estonian Painter at the Courts of Renaissance Europe hrsg. von John Oliver Hand, Greta Koppel (Washington: National Gallery of Art, 2017), 27-37. 
Handwerkerämter in Reval vereinte..$^{49}$ Über seine Tätigkeiten in Reval und seine Werke wissen wir nur anhand von schriftlichen Quellen, wenn wir die durchaus gerechtfertigte Annahme beiseite lassen, er hätte die Außenflügel des Passionretabels ${ }^{50}$ übermalt, das zuerst in der Werkstatt von Adriaen Isenbrandt oder Albert Cornelis entstanden war. ${ }^{51}$ Für unseren Zusammenhang ist das Material des Rosenkranzes in den Händen des Donators von Interesse. Mit seiner orangenen oder rötlichen Farbe, würde das Material der Koralle hier evoziert, dabei verfügt es über einen sehr warmen Farbton, bei dem auch Bernstein erwogen werden sollte. Bei einem Portrait aus Reval würde dies viel Sinn machen, wie unten gezeigt werden soll. Damit würde dieses kleine Detail noch einmal die Annahme, das Portrait stamme aus Reval und wäre von Michel Sittow gefertigt worden, bekräftigen.

\section{DIE ROSENKRÄNZE ALS FROMME SPENDEN IM SPÄTMITTELALTERLICHEN REVAL}

In den Revaler Quellen gibt es zahlreiche Erwähnungen von Rosenkränzen, die teilweise in Güterverzeichnissen der Kirchen auftreten oder mit einem Testament von Revaler Männer oder Frauen vererbt oder einer Kirche gespendet wurden.

Zum Beispiel spendete ein reicher Kaufmann und Mitglied der Bruderschaft der Schwarzenhäupter Hans Bouwer (gest. 1519) in seinem Testament der Nikolaikirche für die Statuen von der Heiligen Anna und des Heiligen Nikolaus. ${ }^{52}$ Die Skulptur der Anna (diese Heilige wurde von Bouwer besonders verehrt) sollte mit

49 Anu Mänd, „Michel Sittow and Reval (Tallinn): A New Look at Records in the Tallinn City Archives", Michel Sittow 1468-1525: the Artist connecting Estonia with Southern Netherlands, hrsg. von Tiina Abel (Tallinn: Eesti Kunstimuuseum, 2001), 9; Anu Mänd, „The City of Tallin
or Reval in the Late Middle Ages and Renaissance”, Michel Sittow. Estonian Painter at the Courts of Renaissance Europe, hrsg. von John Oliver Hand, Greta Koppel (Washington: National Gallery of Art, 2017), 24

50 Werkstatt von Adriaen Isenbrant (Übermalungen der Außenflügel Michel Sittow?). Um 510-1520. Öl auf Holz. Flügel: $189 \times 94 \mathrm{~cm}, 189 \times 96 \mathrm{~cm}$. Estnisches Kunstmuseum - Niguliste Museum

51 Greta Koppel, „The Riddle of Michel Sittow's art”, Michel Sittow. Estonian Painter at the Courts of Renaissance Europe, 4.

52 Anu Mänd, „Hans Bouwer, kaupmees“, Kümme keskaegset tallinlast, hrsg. von Tiina Kala, Juhan Kreem, Anu Mänd (Tallinn: Varrak 2016), 85. einen Rosenkranz aus Silber ausgestattet werden. ${ }^{53}$ Dass war nur ein Teil der Spenden des Hans Bouwer, der einen großen siebenarmigen Leuchter für die Nikolaikirche ${ }^{54}$ in Lübeck gießen ließ und auch die anderen Kirchen und Klöster in seinem Testament miteinbezog und mit Spenden ausstattete (insgesamt hatte er über 7.000 rigische Mark zu vergeben, ein wahres Vermögen). ${ }^{55}$ Zur Verehrung der Heiligen Anna war noch das Messelesen gedacht, wofür Bouwer seine gesamten Immobilien spendete und noch zusätzlich Geld. ${ }^{56}$

Ein anderes Beispiel liefert das Testament einer Frau, Katherina aus dem Jahre 1519, der Witwe des Malers und Bildschnitzers Jurgen Dreger. ${ }^{57}$ Sie vererbt sowohl Kleidung, Schmuck, Bettwäsche und Küchenaustattung, doch daneben spendete sie auch den Kirchen, wahrscheinlich die von ihrem vestorbenen Mann nachgelassenen Skulpturen..$^{58}$ Ihre Tochter erhielt ihren gesamten Silberschmuck, wie auch ihre Kopfzier, Ketten mit dem Heiligen Georg und ein Agnus Dei, Gürtel und Silberknöpfe. ${ }^{59}$ Darunter befand sich auch eine Schnur aus Korallen mit zehn Silbersteinen und ein kleiner Rosenkranz aus Silber. ${ }^{60}$ Ihrem Ehemann gehörte ein Rosenkranz aus Jaspis, den sie seinem Bruder Benedictus vererbte, und ihrem eigenen Bruder überließ sie einen Rosenkranz aus Bernstein. ${ }^{61}$

Zahlreiche Informationen liefern die Quellen in Form von Güterverzeichnissen von Kirchen und Klösterm. Zum Beispiel gehörten dem Johannishospital im Jahre 1448 drei Rosenkränze (sog. Veftich - aus 50 Perlen bestehende Gebetsketten). ${ }^{62}$ In der Heilig-Geist-

53 Mänd, „Hans Bouwer, kaupmees“, 85.

54 Siebenarmige Leuchter. Lübeck 1519. Messing. Höhe: 4 m. Estnisches Kunstmuseum Niguliste Museum.

55 Mänd, „Hans Bouwer, kaupmees“, 78-88.

56 Ibidem, 85

57 Tallinna ajaloo lugemik. Dokumente 13.-20. sajandini, hrsg. von Tiina Kala et al., Tallinna Linnaarhiivi Toimetised Nr. 14 (Tallinn: Tallinna Linnaarhiiv, 2015), 94.

58 Ibidem, 94-96.

59 Ibidem, 95.

60 Ibidem.

61 Ibidem, 96.

62 Güterverzeichnis des Johannishospitals von 1448. Publiziert in: Liv-, Est-und Kurländisches Urkundenbuch. Bd. X. 1444-1449, hrsg. von P. Schwartz (Aalen: Scientia Verlag, 1981), Nr. 528 Publiziert und übersetzt in: Anu Mänd, Kirikute hõbevara. Altaririistad keskaegsel Liivimaa Eesti Kirikute sisustus I (Tallinn: Muinsuskaitseamet, 2008), 203. 
Kirche befanden sich ein Rosenkranz aus Korallen mit Silbersteinen und mit dem Agnus Dei, ein Rosenkranz aus Bernstein mit einigen Silbersteinen und noch andere Rosenkränze aus Koralle. ${ }^{63}$ In der Dominikanerkirche werden mehrmals Rosenkränze aus Koralle ${ }^{64}$ und aus Silber ${ }^{65}$ erwähnt. Einige von diesen Gebetsperlen waren für die Statuen der Gottesmutter ${ }^{66}$ und der Heiligen Anna sowie der Heiligen Katharina gedacht. ${ }^{67}$ Wir wissen, dass das Schwarzenhaupt Hinrick Grymmolt einen Rosenkranz aus Korallen und Silber, das für die Silberstatute der Gottesmutter in der Domikanerkirche St. Katharinen gedacht war, gespendet hat. ${ }^{68}$ Diese Informationen geben den Kontext für die Wiedergabe des Rosenkranzes auf dem Revaler Retabel des Meisters der Legende der Hl. Lucia wieder.

\section{DER ROSENKRANZ IN DEN HÄNDEN EINES KAUFMANNS AUF DEM REVALER RETABEL}

Laut Ravo Reidna kann das Material des Rosenkranzes als Bernstein ${ }^{69}$ identifiziert werden. ${ }^{70}$ Beim Revaler Retabel beginnt und endet der Rosenkranz in offener Form mit blauen Seidenquasten, an beiden Enden befinden sich eine große Paternoster-Perle bzw. ein Abschlussobjekte sind sichtbar, die vermutlich zwei Sätze von zehn Ave-Maria-Perlen umrahtmen. Die Gebetsschnur verfügt über eine

63 Güterverzeichnis der Heilig-Geist-Kirche von 1532. Stadtarchiv Tallinn (Tallinna Linnaarhiiv, TLA) 230-1-B1 5, 1r-2r; Publiziert und übersetzt in: Mänd, Kirikute hõbevara, 207. 64 Güterverzeichnis der Revaler Schwarzenhäupter in der Dominikanerkirche von 1495. TLA, 87-1-95. Publiziert in: Liv-, Est- und Kurländisches Urkundenbuch. 2. Abt. Bd. I. Ende Mai 1494-1500, hrsg. von Leonid Arbusow (Aalen: Scientia Verlag, 1981), Nr. 106: Publiziert und übersezt in: Mänd, Kirikute hõbevara, 218

65 Das Eigentum der Revaler Schwarzenhäupter, das 1524 aus der Dominikanerkirche entfernt wurde. TLA, 87-1-101; publiziert und übersetzt in: Mänd, Kirikute hõbevara, 223.

66 Güterverzeichnis der Dominikanerkirche um 1495. TLA, 87-1-95; publiziert und übersetz in: Mänd, Kirikute hõbevara, 218

67 Von der Dominikanerkirche 1524 und 1525 konfiskierte Güter. TLA, 230-1-Bk 3, 67r publiziert und übersetzt in: Mänd, Kirikute hõbevara, 223-224.

68 Anordnungen des Altarvorstehers der Bruderschaft der Schwarzenhäupter Euert van der Schuren für die Beleuchtung der Altäre und Skulpturen in der Dominikanerkirche. TLA, 87-193. Publiziert und übersetzt in: Tallinna ajaloo lugemik, 86.

69 Ravo Reidna, Tallinna Mustpeade vennaskonna Maarja altar (Tallinn: Eesti Kunstimuuseum 1995), 4.

70 Man muss dennoch bei dieser Beurteilung vorsichtig sein, weil der Firnis den Eindruck der Farbe stark beeinträchtigen kann. Die Kombination von gelben Perlen mit blauen Quasten könnte aber aus künstlerischer Sicht einen gewollten Kontrast der Komplementärfarben erzeugen.

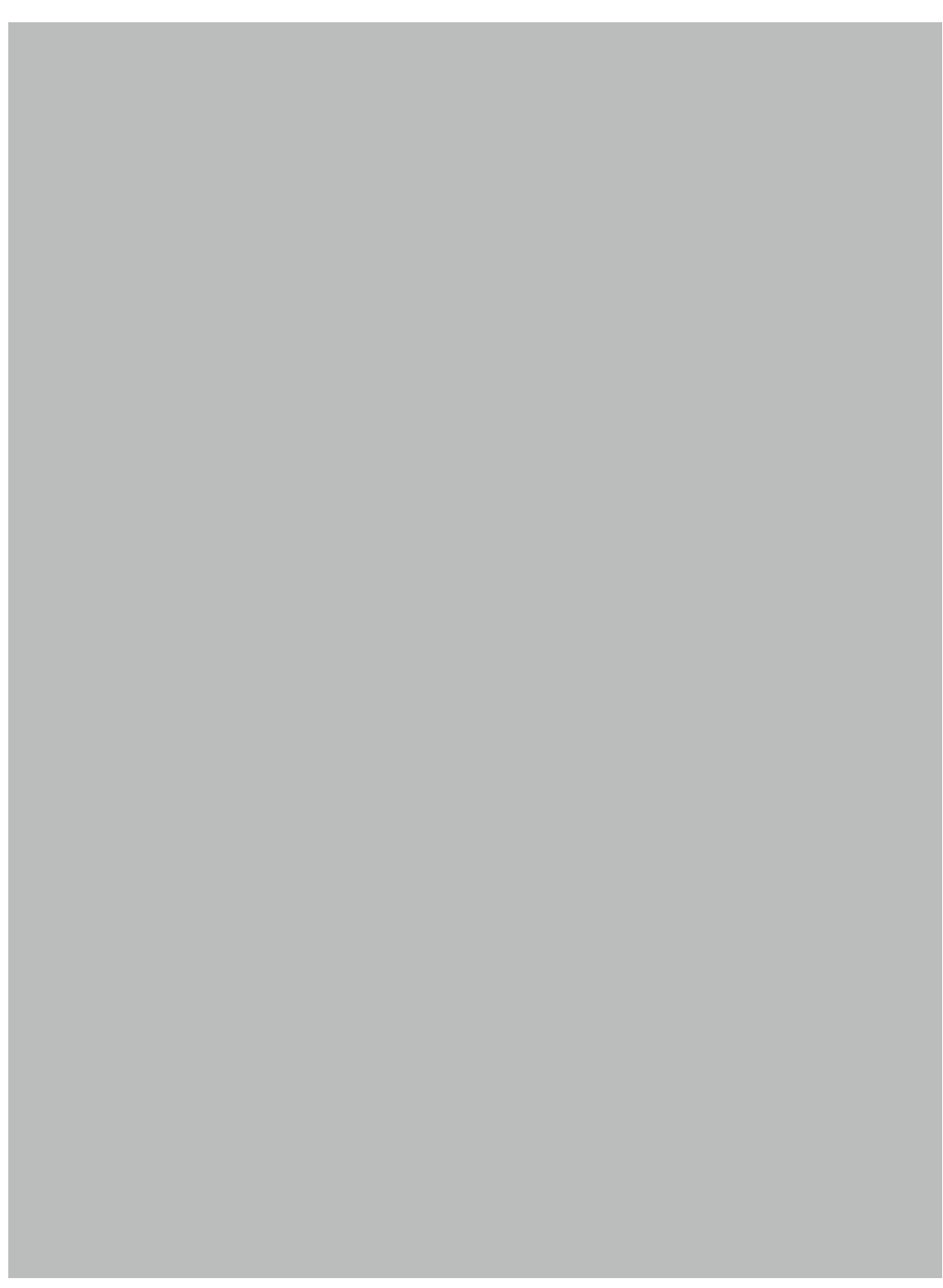

ABB. 8. JAN VAN EYCK. ARNOLFINI DOPPELTPORTRAIT. 1434. ÖL AUF HOLZ. $82,2 \times 60$ CM THE NATIONAL GALLERY, LONDON. 
große Verwandtschaft mit dem Rosenkranz auf dem Arnolfini Portrait ${ }^{71}$ des Jan van Eyck (Abb. 8). Der aus wohl 27 Ave-MariaPerlen, zwei abschließenden Paternoster-Perlen und grünlich blauen Seidenquasten bestehende Rosenkranz aus Kristall hängt hinter den Donatoren, an der Wand neben dem Spiegel mit Passionszenen. Die Anzahl der Perlen und ihre Aufreihung scheint weder bei dem Arnolfini Portrait noch beim Revaler Retabel von Belang zu sein. Es kann argumentiert werden, dass hier die Einhaltung der formellen Elemente eines Zehners oder Rosenkranzes nicht angestrebt worden war und die Aufreihung wie auch die Reihenfolge der Perlen deshalb beliebig blieb. Ein ähnlicher Rosenkranz schmückt den Gürtel der Heiligen Apollonia auf dem Brüsseler Retabel ${ }^{72}$ des Meisters der Legende der Hl. Lucia, er ist aber sehr gut erkennbar aus Kristall und mit goldenen Quasten geschmückt.

Das Arnolfini Doppeltportrait verdient in unserem Zusammenhang eine besondere Aufmerksamkeit, auch weil dessen Maler, Jan van Eyck, eine Schlüsselfigur zum Verständnis des Retabels ist. Die Fülle an Textilien und anderen Erzeugnissen orientiert sich an seinem, in den damaligen Brügger Kirchen gut zugänglichem Schaffen, auch wenn die malerische Genauigkeit des „Königs der Maler“ von dem Meister der Legende der Hl. Lucia zu Gunsten der Material- und Zeitersparnis aufgegeben wurde. Dem Meister der Legende der Hl. Lucia ist keine derart detaillierte Darstellung von Textilien zueigen, die Jan van Eycks CEuvre charakterisiert. Hier könnte von einer Banalisierung des eyckischen Erbes die Rede sein, doch im Rahmen des Revaler Retabels erscheinen auch markante Abweichungen. Die wichtigsten Ausnahmen bilden im Rahmen des Retabels vorrangig all jene Elemente, die einen diminutiven Charakter haben. Dazu gehören Goldschmiedearbeiten, goldene Borten, Buchschließen und Buchverschlüsse und auch der Rosenkranz. Ganz im eyckischen Geiste wird hier das durchsichtige Material und die darauf tänzelnden Lichtreflexe und das Schimmern der Perlen, wenn auch nicht mit einem vergleichbaren malerischen Können, zur Geltung gebracht.

71 Jan van Eyck. Arnolfini Doppeltportrait, 1434, Ö1 auf Holz, 82,2 ×60 cm, National Gallery, London.

72 Meister der Legende der H1. Lucia. Madonna und Kind unter weiblichen Heiligen. Um $1475-1480.107,8 \times 171 \mathrm{~cm}$. Koninklijke Musea voor Schone Kunsten van België; Musées royaux des Beaux-Arts de Belgique, Brüssel.

\section{DER ROSENKRANZ ALS EIN HINWEIS AUF DEN BESTIMMUNGSORT UND URSPRUNGSORT}

Bei der Miteinbeziehung eines Rosenkranzes in den Händen des Donators, ist der wahrscheinliche Aufstellungsort des Retabels - die St. Katharinenkirche der Dominikaner - von Interesse. Vornehmlich der Dominikanerorden leistete eine wesentliche Rolle bei der Verbreitung und Förderung des Rosenkranzes und Rosenkranzgebets, dessen Hintergrund der Glaube war, dass der Heilige Dominikus selbst der Stifter des Rosenkranzes gewesen sei, dem dies von der Muttergottes, offenbart worden war. ${ }^{73}$ Diese Legende wurde von dem bretonischen Dominikaner, Pater Alanus de Rupe OP, in Umlauf gebracht, deren Wirken zu verdanken ist, dass das Rosenkranzgebet auch ein Laiengebet wurde ${ }^{74}$. Auch die Gründungen der Rosenkranzbruderschaften sind erstmals von ihm initiiert worden. Für unseren Zusammenhang kann übernommen werden, dass der Rosenkranz in einer Szene erscheint, die einen Bezug zu den Dominikanern hat. In der Szene der doppelten Fürbitte erscheinen die Gottesmutter, der Schmerzensmann und Johannes der Täufer, die vor dem thronenden Gottvater für die 30 knienden Kaufleute Fürbitte leisten. Die Szene findet Ihren ikonographischen Ursprung in einem mittelalterlichen typologischen Manuskript, in dem sogenannten Speculum Humanae Salvationis oder dem Heilsspiegel. Der Verfasser der Handschrift blieb anonym, doch kann ein gelehrter Geistlicher, wahrscheinlich ein Dominikanermönch, als Autor vermutet werden. ${ }^{75}$ Die Wahl dieser Szene hat die These hervorgebracht, die Dominikaner hätten bei der Gestaltung

73 Andreas Heinz, „Die Entstehung des Leben-Jesu Rosenkranzes“, Der Rosenkranz: Andacht Geschichte, Kunst [Ausstellung „,Zeitinseln - Ankerperlen, Geschichten um den Rosenkranz Museum Bruder Klaus Sachseln 25. Mai bis 26. Oktober 2003] (Bern: Benteli, 2003), 23.

74 Heinz, „Die Entstehung des Leben-Jesu Rosenkranzes“, 38-39.

75 Manuela Niesner, Das Speculum humanae salvationis der Stiftsbibliothek Kremsmünster: Edition der mittelhochdeutschen Versübersetzung und Studien zum Verhältnis von Bild und Text. Schriftenreihe Pictura et Poesis, Bd 8 (Köln; Weimar; Wien: Böhlau, 1995), 7, 11-12, 25 ; Kerttu Palginõmm, Lucia legendi meistri Tallinna retaabli topelteestkostestseen illumineerijate ja maalikunstnike suhete taustal. Unveröffentliche Masterarbeit (Universität Tartu, 2010). 
des ikonographischen Programms als Vermittler gedient. ${ }^{76}$ Die Fürbitteszene selbst ist mit dem Rosenkranzgebet eng verbunden, ob durch die Gottesmutter als Schutzmantelmadonna oder durch das meditative Gebet über die fünf Wundmale Jesu Christi. ${ }^{77}$ Die Fürbitte richtet sich oft gegen Krankheiten, Krieg und die Pest. ${ }^{78}$ Betrachtet man die mit dem Rosenkranz eng verbundene Fürbitteszene und die Bedeutung des Rosenkranzes für die Dominikaner, kann damit deduziert werden, dass dieses Detail Bezug auf den Bestimmungsort, also den klösterlichen Kontext, nehmen könnte. ${ }^{79}$

Der Rosenkranz kann aber auch anders gedeutet werden, nämlich als ein Hinweis auf den niederländischen Kontext. Das Rosenkranzgebet hat Bezüge zu mittelalterlichen niederländischen Marienlegenden. Der Legende nach, beten drei Schwestern den Rosenkranz, damit die Gottesmutter Maria einen feinen Mantel, Kleid und Unterkleid und "Juwelen" sowie Kopfschmuck und zusätzlich Schuhe erhält. ${ }^{80}$ Der Legende nach müssen sie sich besonders bemühen und den Rosekranz beten, um der Gottesmutter feine Kleidungen zu bieten. Am Ende würdigen Sie die Gottesmutter, die Heilige Katharina und die Heilige Agnes mit vornehmen Kleidern und Schmuck und gewinnen im Anschluss die ewige Krone im Himmel. ${ }^{81}$

Auf dem Revaler Retabel erscheint die Gottesmutter gleich dreimal -im geschlossenen Zustand in der Verkündigungszene in grau im grau gemalten Kleid und Mantel, in der Fürbitteszene mit einem goldbestickten blauen Mantel und in der allerfestlichen Szene als Himmelskönigin mit einer mit Edelsteinen verzierten Lilienkrone, im roten pelzbesetzten Kleid mit einem goldbestickten und mit

76 Mänd, „Lucia legendi meister identifitseeritud?“, 188; Mänd, „The Altarpiece of the Virgin Mary of the Confraternity of the Black Heads in Tallinn“, 51; Lehti Mairike Keelmann, „Success, Socal and Regional Saint Cult's"s, Saints as Intercessors between the Wealthy and the Divine. Art and Hagiography among the Medieval Merchant Classes, hrsg. von Emily Kelley, Cynthia Turner Camp (London; New York: Routledge, 2019), 223.

77 As-Vijvers, ,Weaving Mary's Chaplet“, 62-63.

78 As-Vijevers, „Weaving Mary’s Chaplet“, 64; Lehti Mairike Keelmann, Bachelors Bridging the Baltic: The Artistic Ambitions of the Tallinn Brotherhood of the Black Heads. Dissertation (Vorläufiges Manuskript) (University of Michigan, 2017), 189.

79 Keelmann, Bachelors Bridging the Baltic, 194, 197.

80 As-Vijevers, ,Weaving Mary’s Chaplet“, 41

81 Ibidem, 42
Edelsteinen besetzten blauen Unterkleid und Mantel. So wie in der Legende von dem intensiven Rosenkranzgebet der drei Schwestern, die um reiche Gewänder für die heiligen Personen beten und es davon abhängt, welche Gewänder diese erhalten, so steigert sich der Materialwert der Szenen des Retabels mit jedem weiteren Öffnen. In der Legende wird die Herstellung der reichen Gewänder mit dem Beten des Rosenkranzes gleichgesetzt. Dieser Kontext mag dem niederländischen Künstler bekannt gewesen sein und könnte das Vorwissen des Betrachters vor dem Retabel aktiviert haben. Die Legenden waren in der Volkssprache niedergeschrieben und auch mündlich verbreitet. ${ }^{82}$ Über die Verbreitung der Legende in Livland liegen keine Quellen vor, doch muss mit grenzüberschreitenden Texten und Ideentausausch gerechnet werden, so wie wir auch vermuten müssen, dass das Speculum Humanae Salvationis einmal in der Bibliothek der Dominikaner vorhanden gewesen war. ${ }^{83}$ Weiterhin zählte Livland damals wie der Ostseeraum, Nord- und Nordostdeutschland sowie die Niederlande zum mittelniederdeutschen Kulturraum.

\section{DER ROSENKRANZ ALS EIN INSTRUMENT DES GEBETS}

Das Gebet des Donators richtet sich an die Fürbittenden oder dezidiert an die Gottesmutter. Hervorzuheben ist, dass es sich beim Rosenkranz nicht um ein passives Attribut handelt, sondern von einer aktiven Nutzung der Gebetskette gesprochen werden kann. Auf dem Retabel wird ein Moment dargestellt, als der „Kaufmann“ gerade eine Perle zwischen seinen Fingern hält und das Bittgebet "Gegrüßest seist du, Maria...", also das Ave-Maria, betet und über ein Ereignis des Heilsgeschehens meditiert. ${ }^{84}$ Bei einem sehr auf Maria, die Muttergottes, ausgerichteten ikonographischen Programm ist dies inhaltlich motiviert. Auch der von Textilien gestaltete nicht konkretisierte Raum kann einen kontemplativen Charakter der Situation hervorrufen. Es kann auch vermutet werden, dass der meditierende Donator das Geschehen um ihn herum vor seinen

82 As-Vijevers, ,Weaving Mary’s Chaplet“, 41 .

83 Man geht davon aus, dass in jeder wichtigen Bibliothek ein Exemplar vorhanden war. Siehe: Adrian Wilson, A medieval mirror: Speculum Humanae Salvationis 1324-1500 (Berkeley [u.a.]: University of California Press, 1984), 24.

84 Keelmann, Bachelors Bridging the Baltic, 197. 
geistigen Auge wahrnimmt - eine Praxis, die in der zeitgenössischen Andachtsliteratur bezeugt ist. ${ }^{85}$ Eine zusätzliche Bedeutungsebene kann noch hinzugefügt werden. Das Retabel stand einst auf einen Bruderschaftsaltar in der Dominikanerkirche, vor dem die Mönche sowohl Messe gelesen als auch Fürbitte geleistet haben So richtet sich das Gebet der Mönche für die Großen Gilde und die Bruderschaft der Schwarzenhäupter, die in Form der gemalten Mitglieder gegenwärtig sind, an die Fürbittenden Maria, Jesus Christus und Johannes den Täufer. Die Aufgabe des Predigerordens, für die Gilde und die Bruderschaft zu beten, könnte die Wahl der Szene initiiert haben und mag auf die Vermittlerrolle der Dominikaner Bezug nehmen. ${ }^{86}$

\section{DER ROSENKRANZ ALS EIN LUXUSGUT}

Der Rosenkranz auf dem Revaler Retabel hat einen vielschichtigen Charakter. Zum einen zeugt er von der Frömmigkeit der Kaufleute und von der aktiven Beteiligung am Gebet, zum anderen handelt es sich um einen Luxusgegenstand, den wenige Auserwählte sich leisten konnten. ${ }^{87}$ Auch beim Arnolfini Portrait, das einen italienischen Kaufmann mit seiner Frau darstellt, deuten verschiedene Details wie der Rosenkranz, aber genauer noch die Orangen auf der Fensterbank oder die luxuriösen Textilien auf die Rolle Arnolfinis als Vermittler von Luxusgüter am Hof und auf seine eigene wohlhabende Position im alltäglichen Leben hin. So wie es Craig Harbison formuliert hat waren die dargestellten Apfelsinen auf den Fensterbank eigentlich nur für den Fürsten zugänglich, eine Darstellung, die den Eindruck vermittelte, dass die Familie Arnolfini über große Ressourcen verfügte. ${ }^{88}$ Auch hier muss wieder die Brügger Vorstellung von einem Kaufmann betont werden. Wenn der Meister der Legende der Hl. Lucia für Kaufleute malte, so hatte er einen bestimmten idealen Kaufmann

85 Rothstein, Sight and Spirituality in Early Netherlandish Painting, 95-96; Keelmann, Bachelors Bridging the Baltic, 198.

86 Keelmann, Bachelors Bridging the Baltic, 194, 197.

87 Lehti Mairike Keelmann, „Amber Rosaries, Baltic Furs, and Persian Carpets: The Tallinn Mary Altarpiece as an Object of Hanseatic Conspicuous Consumption?", Beyond the Sea:

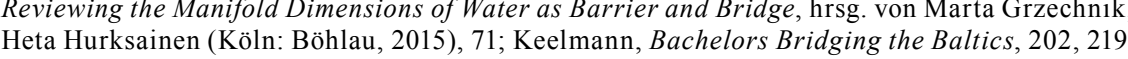
88 Harbison, Jan van Eyck, 37. vor Augen, dessen Wünsche er zu berücksichtigen versuchte. So versieht Jan van Eyck den Kaufmann Arnolfini auf seinen Portraits mit Luxusgegenständen. Auf diese Weise möchte der Meister der Legende der Hl. Lucia die Hansekaufleute zufriedenstellen, indem er das malt, womit er meint seiner Donatoren am besten zu präsentieren. Das Ideal der mittelalterlichen Welt war Bescheidenheit und Armut, doch ein Luxusprodukt wie der Rosenkranz aus kostbaren Material war dadurch legitimiert, dass es ein religiöses Attribut war, gedacht für die Andacht und das Lob Gottes. ${ }^{89}$

Bernstein galt als eines der international bekanntesten Produkte der östlichen Ostseeküste. Es wurde auch früher vermutet, dass die Spender dieses „einheimische Produkt ${ }^{\prime 90}$ auf dem Retabel dargestellt sehen wollten. Im Spätmittelalter monopolisierte der Deutsche Orden in Preußen den Handel mit Bernstein: das gesamte von seinen Untertanen gefundenes Material sollte dem Bernsteinmeister übergeben werden. ${ }^{91}$ Der Bernsteinmeister schickte das Bernstein weiter an den Großschäffer von Königsberg, dessen Ziel es war, das Rohmaterial für den besten Preis weiterzuverkaufen. ${ }^{92}$ Auch wenn die livländischen Kaufleute wohl kaum mit Bernstein gehandelt haben können wir davon ausgehen, dass der Meister der Legende der $\mathrm{Hl}$. Lucia die Region als Deutschordensgebiet insgesamt mit diesem Material in Verbindung brachte und es dementsprechend auch in den Händen seiner Donatoren darstellt. ${ }^{93}$ So wie die meisten Güter Brügge als Umschlagplatz der Waren und Wirtschaftsmetropole definierten, so zeugten bestimmte Waren im Rahmen des Retabels von dem Ursprungsgebiet der Donatoren. Dabei muss im Auge behalten werden, dass, auch wenn der Bernstein aus dem Territorium des Deutschen Ordens kam, die wichtigsten Bernsteindreher sich

89 As-Vijevers, „Weaving Mary's Chaplet“, 71.

90 Reidna, Tallinna Mustpeade vennaskonna Maarja altar, 4; Lehti Mairike Keelmann, „Amber Rosaries, Baltic Furs, and Persian Carpets: The Tallinn Mary Altarpiece as an Object of Hanseatic Conspicuous Consumption?" Beyond the Sea: Reviewing the Manifold Dimensions of Water as Barrier and Bridge, hrsg. von Marta Grzechnik, Heta Hurksainen (Köln: Böhlau, 2015), 71. 91 Carsten Jahnke, „The Baltic Trade“, A Companion the Hanseatic League, hrsg. von Donald Harreld (Leiden: Brill, 2015), 208

92 Jahnke, „The Baltic Trade“, 208.

93 Kerttu Palginõmm, „Luxusartikel auf dem Revaler Retabel des Meisters der Lucialegende als eine Einladung in die Stadt Brügge“", Baltic Journal of Art History, 3 (2012), 11; Keelmann, Bachelors Bridging the Baltics, 201. 
sowohl in Brügge als auch in Lübeck befanden. ${ }^{94}$ Die Lübecker paternostermakers produzierten Rosenkränze für die Märkte in Venedig, Nürnberg und Frankfurt, sollten aber eine festgelegte Anzahl der Rosenkränze an den Deutschen Orden kostenlos als Gegenleistung für das Rohrmaterial übergeben und produzierten ansonsten für Venedig und Spanien. ${ }^{95}$ Zum Beispiel vermittelte Hildebrandt von Veckinchusen Rosenkränze aus Bernstein nach Venedig. ${ }^{96}$ Später nach dem Dreizehnjährigen Krieg (auch Preußischer Städtekrieg genannt, 1454-1466) gingen die westlichen Gebiete des Deutschen Orden (darunter auch Danzig) in Preußen an die Hände des polnischen Königs Kasimir VI. über. Darauf haben die Städte teils das Handel übernommen, so dass auch lokale Bernsteindreher, wie in Danzig 1477, begründet wurden. ${ }^{97}$ Dieses war verheerend für die Bernsteindreher von Brügge, so dass das Zunft schon im Jahr 1498, also fünf Jahre nach dem Ankunft des Retabels, aufgehört hatte zu bestehen. ${ }^{98}$ Der Bernsteinrosenkranz in den Händen des Donatoren diente als ein Zeichen der früheren Blüte der Hansestad Brügge und der jetzigen Situation. In der Gesamtheit der Luxusgüter gesehen, wobei der Betrachter sowohl Güter dargestellt sieht, die zum Beispiel sowohl aus Osten als auch aus dem Westen kommen, kann dies als ein weiteres Indiz für das glückliche Weiterbestehen der Handelsbeziehungen interpretiert werden - dieser Eindruck sollte vermittelt werden. In diesem Sinne können auch die hansischen Wirtschaftsbeziehungen, wenn auch nicht intendiert, auf dem Retabel erblickt werden.

\section{ZUSAMMENFASSUNG}

Der Rosenkranz in den Händen des Donatoren auf dem Brügger Retabel des Meisters der Legende der Hl. Lucia bringt eine mögliche Interpretationskette mit sich, die sowohl für den Revaler wie auch

94 Jan Albert Van Houtte, ,Ambernijverheid en paternostermakers te Brugge gedurende de XIVe en XVe eeuw“, Essays on Medieval and Early Modern Economy and Society, hrsg. von Jan Albert van Houtte (Leuven: Leuven University Press, 1977), 80.

95 Jahnke, „The Baltic Trade“, 210.

96 Van Houtte, „Ambernijeverheid en pasternostemakers te Brugge gedurende de XIVe en XVe eeuw", 57.

97 Jahnke, „The Baltic Trade“, 210.

98 Van Houtte, „Ambernijverheid en paternostermakers te Brugge gedurende de XIVe en XVe eeuw", 80 . für den Brügger Kontext aufschlussreich sein kann. Das als eine devote Haltung des Kaufmanns gedeutete Gebetsinstrument ist gleichzeitig auch ein Luxusgut, dessen Material sowohl als Bernstein wie auch als Kristall angesehen werden kann. Das „wahre Material“ des Luxusguts, womöglich Bernstein, ist dabei nicht unwichtig, denn es deutet auf das Gebiet der östlichen Ostseeküste hin, wo man mit dem Material handelte. Auf einem Brügger Retabel dient es als eine Referenz auf die Auftraggeber. Die Ausführung und das malerische Können, das von dem anonymen Maler bei der Umgestaltung des Rosenkranzes hier demonstriert wird, nimmt Bezug auf die lange Künstlertradition von Brügge, wo die Rosenkränze aus Bernstein in großer Zahl bis Ende des 15. Jahrhunderts produziert wurden.

Die Miteinbeziehung des Rosenkranzes ist einleuchtend, wenn man den Bestimmungsort des Retabels, also die. St. Katharinenkirche der Dominikaner, betrachtet. Die Dominikaner waren die Verbreiter des Rosenkranzgebets gewesen, so wie die Revaler Brüder für die Große Gilde und die Bruderschaft der Schwarzenhäupter Fürbitte leisteten. Aus der Perspektive des Meisters der Legende der Hl. Lucia betrachtet, war aber der Rosenkranz in den mittelalterlichen Marienlegenden mit der Anfertigung von kostbarer Kleidung für die Gottesmutter verbunden. Das Revaler Retabel ist besonders in der Dichte der dargestellten Luxusgüter ohne Parallele - dabei spielen besonders Seiden- und Brokatstoffe eine große Rolle. Auch die Gottesmutter erscheint gleich dreimal in kostbaren Kleidern.

Diese Überlegungen führen uns auf die Frage nach der Initiative des Auftraggebers und der des Malers hin. Dies erzeugt auch die Frage, ob der Rosenkranz hier nicht überinterpretiert wird. Wir können uns gut vorstellen, dass die Initiative des Darstellens, entweder aus Brügge oder aus Reval kommt. Doch die Konnotationen, die damit verbunden sind, können nur zum gewissen Grade geteilt werden. Einem Revaler Kaufmann kann die Marienlegende unbekannt sein doch in den niederländischen Kontext gesetzt wird sie verständlich Der Maler mochte in Unwissen darüber gewesen sein, ob das Retabel für eine Dominikanerkirche bestimmt war, doch er weiß, dass das Rohrmaterial für die Bernsteindreher von Brügge „aus dem Osten“ kommt. Zusätzlich zu der wirtschaftlichen Bedeutung des Rosenkranzes, möchte der Maler den Kaufmann als einen sehr 
devoten Christen präsentieren. Damit geht er auf die vermeintlichen Wünsche der Auftraggeber ein. Ungeachtet des ersten Impulses für eine Wiedergabe des Rosenkranzes, auch wenn dies von Anfang an als nichts anderes als ein einfaches Gebetsinstrument intendiert gewesen war, gewinnt es trotz allem eine vielschichtige Bedeutung vor Ort, in der Hansestadt Reval, in der Dominikanerkirche der Stadt, auf dem Bruderschaftsaltar in der Kirche, in den Händen eines Kaufmanns.

Kerttu Palginõmm: The Attributes of Merchants - a Glance at the Tallinn (Reval) Altarpiece of the St. Mary's Altar of the Brotherhood of the Black Heads

Keywords: material culture studies; Burgundian Netherlands; Hanseatic networks; Netherlandish art; Flemish altarpiece; ROSARY; AMBER

\section{SUMMARY}

The article focuses on the Tallinn (Reval) altarpiece of St. Mary's altar of the Brotherhood of the Blackheads, attributed to the Bruges Master of the Legend of St. Lucy, which arrived in the late medieval city in 1493. The altarpiece is noteworthy for its very dense network of luxurious details like silk, velvet and gold cloth from Italy, ceramics from Spain, tapestries from Netherlands and an oriental carpet from Anatolia. Early Netherlandish painting is notable for the very detailed, texture and light-sensitive rendering of everyday and luxury objects, from which the works of Jan van Eyck are the best examples.
The altarpiece depicts thirty merchants in colourful woollen clothes carrying attributes that signify their trades, like pouches and signet rings. These display their power and authority, status and wealth, identifying them as Hanseatic merchants trading in textiles and other goods. One of the merchants is holding a crystal or amber rosary - the material of the rosary cannot be determined precisely due to the condition of the altarpiece (the varnish may influence the nuances of the colour palette). Several Netherlandish altarpieces feature rosaries like the Merode triptych of the Master of Flemalle (Robert Campin?) and the Arnolfini Portrait of Jan van Eyck. The rosary in the Arnolfini portrait most closely resembles the one held by the Livonian merchant.

The amber or crystal rosary is laden with meaning. Since the merchant is holding one prayer bead between his fingers, it is being actively used as a devotional object. He is in deep meditation over particular sacred event and praying the Ave Maria. The rosary was especially favoured by the Dominicans - and it carries a special meaning in the case of the Tallinn altarpiece, which was destined for the Dominican church of St. Catherine. Since the monks of the convent assumed the role of intermediaries, they might have exercised their power over the iconography of the altarpiece. The rosary could be pointing to the destined location, but could also be pointing to the place of departure. There was an old Netherlandish legend according to which the Virgin Mary and the saints would be revealed to young women praying the rosary in ever more luxurious attire, the better behaved the girls were, which brings to mind the varied gold, silk and velvet fabrics on the altarpiece.

The material of the rosary is of great importance, because it was a "Baltic product" traded by the Teutonic Order. The raw material was partly traded for the rosaries made in Bruges, or later in Lübeck or Danzig. In 1493, when the altarpiece arrived in Tallinn, the rosary production in Bruges was already in decline. In this way, the rosary becomes the symbol of a continuing successful trading relationships. As a luxury object it is an attribute of position, wealth, but also the devotion of the owner. Written sources from Tallinn feature several rosaries (made of amber among other materials) belonging to merchants and craftsmen, and sometimes, being donated to various Tallinn churches. Was the incorporation initiated by the merchants or by the artist cannot definitely be determined and, thus, results in the question of whether the object might be overinterpreted. The artist may have been unaware of the future location of the altarpiece, and the merchants may have not 
been familiar with the Marian legend. And even if the rosary was only meant to function as a devotional object, it increased in importance in the hands of the merchant once it arrived at the St. Mary's altar of the Brotherhood of the Black Heads, in the Dominican Church of St. Catherine in the Hanseatic city of Tallinn (Reval).

\section{CV}

Kerttu Palginõmm, (PhD candidate of University of Tartu and TU Dortmund University) is a curator and collection-keeper at the Art Museum of Estonia - Niguliste Museum. Her main research areas include the art and material culture of Burgundian Netherlands, with particular interest in luxury goods in Netherlandish painting. She has curated several exhibitions, for example, The Glory of the Cathedral (University of Tartu Museum 2017-2018) and The Power of Things (Art Museum of Estonia - Niguliste Museum 2018-2019). Palginõmm has published articles, edited and co-authored catalogues. 